nerve, but no evidence of tumour or abnormal vasculature. Post-operatively her visual acuity was unchanged.

Review of the literature reveals that this type of pathology is very unusual. Intrachiasmal haematomata have been recognised, some of which are due to chiasmatic gliomas. ${ }^{12}$ Bleeding causing chiasmatic compression has also been reported secondary to cryptic vascular malformations identified at operation $^{34}$ and in patients with clinical evidence of vascular anomalies elsewhere. ${ }^{5}$ Burnbaum et $a l^{6}$ described a patient with a blue-domed haemorrhagic cyst arising extrinsically to the optic nerve and compressing it at its junction with the chiasm. Holt recorded a similar case. ${ }^{7}$ The likely cause of these lesions is haemorrhage into a pre-existing cyst.

The aetiology of our patient's intraneural haematoma is not clear. Maitland et al proposed that most idiopathic intrachiasmatic haematomata are caused by small venous or cryptic vascular malformations, and this would seem to be the most likely explanation with our case. The finding of optic atrophy after only three weeks of symptoms would support this possibility.

RP GREGORY

G KOUTSOUBELIS RFC KERR CBT ADAMS

Department of Neurosurgery, Radcliffe Infirmary,

1 Maitland CG, Abiko S, Hoyt WF, Wilson CB, Okamura $\mathrm{T}$. Chiasmal apoplexy: report four cases. J Neurosurg 1982;56:118-22.

2 Schneider RC, Kriss FC, Falls HF. Prechiasma infarctions associated with intrachiasmal and suprasellar tumours. J Neurosurg 1970;32: 197-208.

3 Roski RA, Gardner JH, Spetzler RF. Intrachiasmatic arteriovenous malformation. Case report. J Neurosurg 1981;54:540-1.

4 Fermaglich J, Kattah J, Manz H. Venous angioma of the optic chiasm. Ann Neurol 1978 4:470-1.

5 Rishede J, Seedorff HH. Spontaneous haematoma of the optic chiasma. Report of a case. Acta Ophthalmol 1974;52:317-22.

6 Burnbaum MD, Harbison JW, Selhorst JB, Young HF. Blue-domed cyst with optic nerve Young HF. Blue-domed cyst with optic nerve
compression. J Neurol Neurosurg Psychiatry compression. $J$

7 Holt $\mathrm{H}$. Cysts of the intracranial portion of the optic nerve. Am J Ophthalmol 1966;61: 1166-70.

Displaced Torkildsen's shunt: an unusual cause of cervical myelopathy

The Torkildsen's shunt procedure involves placing the proximal end of a rubber catheter through a burr hole into the occipital horn of the lateral ventricle, and the distal end through the cisterna magna into the posterolateral cervical gutter, where it is then sutured to the dura." This procedure is nowadays seldom employed due to the high failure rate but complications are rare after the shunt has been functioning for a number of years. ${ }^{2}$ The most frequent complications are infections and the development of a false meningocoele due to leakage of CSF through the cervical dura mater. ${ }^{2}$

A 75 year old male presented after six months of progressive gait unsteadiness and urinary incontinence. In 1964 he developed obstructive hydrocephalus due to a posttraumatic aqueductal stenosis, and was treated with a Torkildsen shunt. He had a

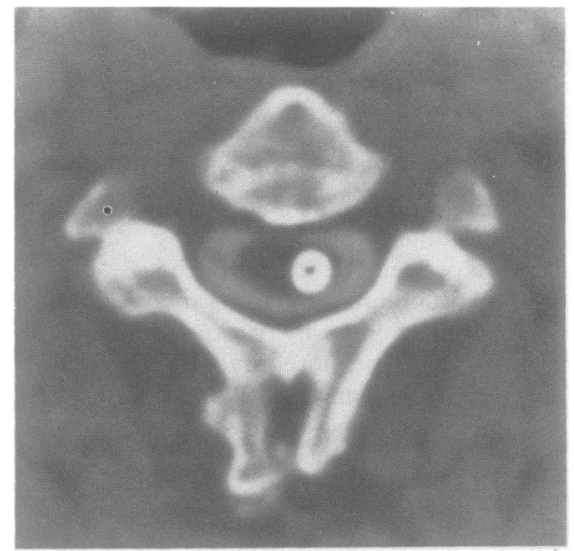

Our case is unusual since the catheter migrated into the dorsal aspect of the cervical cord causing a false syrinx. To our knowledge this late complication has not been reported before, but it should be noted for the patients who still have the ventriculocisternostomy catheter in place.

ALVARO PASCUAL-LEONE ANIL DHUNA

Department of Neurology, University of Minnesota, REYNAL DO CASTII Grand Rapids, Michigan TOM ALA

Department of Neurology,

St Paul Ramsey Medical Center, St Paul, MN

Figure Axial CT through cervical C2 level showing the Torkildsen's shunt tube imbedded into the cord causing a false syrinx.

history of alcohol induced peripheral neuropathy.

On admission he was alert, disoriented to time, and his attention and short term memory were very impaired. The only abnormality noted on his general physical examination was the right parieto-occipital burr hole for the shunt; vital signs were normal. Cranial nerve examination was unremarkable; there was no papilloedema. He had spasticity in all four extremities, marked hyperreflexia in both arms, areflexia in the legs, and bilaterally extensor plantar responses. Sensory examination showed marked vibratory and propioceptive losses in the feet. He had dysmetria and intention tremor of all extremities and his gait was wide based and unsteady.

A cranial CT demonstrated dilatation of the lateral and third ventricles and a right parietal shunt tube terminating in the mid right ventricular body. A temporary ventriculostomy was made and the intracranial pressure readings were never higher than $8 \mathrm{~cm} \mathrm{H}_{2} \mathrm{O}$ over a 48 hour period. A CT myelogram revealed that the distal tip of the shunt tube was within the parenchyma of the upper cervical spinal cord (fig).

The patient had a cervical $\mathrm{C} 1-\mathrm{C} 3$ posterior laminectomy. The shunt tube was found imbedded in the spinal cord; consistent with the CT image. The tube was withdrawn from the cord cavity without difficulty and shown to be draining CSF. It was then shortened, repositioned in the subarachnoid space, and sutured to the dura at C2 level. No intraoperative complications occurred but on the third postoperative day the patient became lethargic and quadriparetic. An emergent CT of the head and cervical spine revealed increased ventricular size and a posterior cervical fluid collection from C2 to C4 that was displacing the cord. Despite the placement of a ventriculo-peritoneal shunt the patient failed to improve and died of a necrotising pneumonitis one month later.

The reported cases of migration of the Torkildsen's catheter describe the tube breaking loose from the dura and burying itself into an extra-arachnoidal position. The CSF then drains into the soft tissue and causes a blind sac in the upper cervical region and the intracranial hypertension reoccurs. This is probably what occurred in our patient on the third post operative day.
1 Scarff JE. Treatment of hydrocephalus: an historical and critical review of methods and results. J Neurol Neurosurg Psychiatry results. ${ }_{1063 ; 26: 1-26 .}$.

2 Torkildsen A. A new palliative operation in cases of inoperable occlusion of the Sylvian aqueduct. Acta Chir Scand 1939;82:117-24.

3 Paine KW, McKissok W. Aqueduct stenosis Clinical aspects, and results of treatment by ventriculocisternostomy (Torkildsen's operation). J Neurosurg 1955;12:127-45.

\section{Guillain-Barré syndrome associated with idiopathic thrombocytopenic purpura}

There is mounting evidence that nerve injury in Guillain-Barre syndrome (GBS) is immunologically mediated, but the roles of cellular and humoral immune mechanisms are uncertain. The association of autoimmune diseases with GBS adds further support to the hypothesis of an autoimmune basis for this disorder. We report a patient who simultaneously experienced GBS and idiopathic thrombocytopenic purpura (ITP) following an upper respiratory tract infection.

This 75 year old woman developed acute progressive quadriparesis two weeks after the start of influenza-like symptoms consisting of fever, sore throat and coryza, which were treated with aspirin and amoxicillin. On examination there was flaccid areflexic quadriplegia (MRC grade 0 to 2), palsies of the right seventh and ninth cranial nerves, and distally diminished sensation in all extremities. Numerous petechiae were observed on the hands, wrists, legs and buccal mucosa. The spleen was not enlarged. Haemoglobin was $13.2 \mathrm{~g} / \mathrm{dl}$ and haematocrit $38 \cdot 8 \%$. The white cell count was $12000 / \mathrm{mm}^{3}$, with a normal differential count. Platelet count was $6000 /$ $\mathrm{mm}^{3}$. The prothrombin time and partial thromboplastin time were normal. Urinalysis was normal except for haematuria. Liver function studies, serum protein electrophoresis, antinuclear antibodies, rheumatoid factor, complement levels and LE test were all negative or normal. An increased number of megakaryocytes was found in an otherwise normal bone marrow examination. Plateletassociated and plasma autoantibodies were investigated by immunofluorescence techniques but failed to demonstrate any immunoreactivity. Prednisone, $60 \mathrm{mg}$ daily, was administered and the platelet count gradually increased to $250000 / \mathrm{mm}^{3}$ on the seventh hospital day. 
On the ninth day, the CSF contained a protein level of $133 \mathrm{mg} / \mathrm{dl}$ and one $1 \mathrm{ym}$ phocyte $/ \mathrm{mm}^{3}$. Electrophysiological study was performed on the twenty first hospital day. Motor nerve conduction of median nerve was $34 \mathrm{~ms}^{-1}$ with a distal motor latency of $4.5 \mathrm{~ms}$ (normal, up to $4 \cdot 2$ ). $\mathrm{F}$ responses were not obtained. Distal sensory nerve conduction velocity of median nerve was $29 \mathrm{~m}^{-1}$, sensory action potential being small $(1.5 \mu \mathrm{V})$. The extensor digitorum brevis muscle was denervated and motor conduction along the peroneal nerve could not be measured. Motor latency from the capitulum fibulae to the tibialis anterior was slightly prolonged, but the $\mathrm{M}$-wave was very decreased $(0.1 \mathrm{mV})$. Electromyography of the deltoid, abductor pollicis brevis, rectus femoris and tibialis anterior muscles revealed a pattern of discrete activity with an increase in mean duration of individual motor unit potentials. Fibrillation potentials and positive waves were only observed in the deltoid muscle. Six months after the onset of illness the patient still had pronounced proximal (MRC 2 to 3 ) and distal (MRC 3 to 4) weakness.

The diagnosis of ITP was strongly supported in our patient by the reduced number of circulating platelets, increased number of megakaryocytes, absence of splenomegaly and the response to prednisone. Specific IgG antibodies binding to defined platelet membrane glycoprotein antigens have been demonstrated in patients with chronic ITP, confirming the autoimmune nature of this disease. ' In acute ITP following a viral infection, the thrombocytopenic factors, be it specific platelet IgM antibodies or immune complexes, have not been defined. Failure to detect antibodies on platelets and in the serum of our patient might be the result of limited sensitivity of the immunofluorescence test. $^{1}$

This patient also fulfilled the diagnostic criteria for GBS. ${ }^{2}$ This is an inflammatory demyelinating neuropathy in which both protein and lipid antigens in peripheral nerve myelin are the target of immune attack, but the pathogenesis is still debatable. Experimental allergic neuritis induced by immunisation with the P2 protein has been considered a disease of cellular immunity and the experimental model of GBS. ${ }^{2}$ Alternatively, during acute-phase illness the serum of patients with GBS contains complementfixing antiperipheral nerve IgM antibodies. These antibodies bind to a neutral glycolipid in the myelin that has yet to be completely identified. ${ }^{3}$ Circulating immune complexes may constitute an additional type of pathogenetic mechanism to produce demyelination.

GBS rarely occurs concurrently with another autoimmune disorder. ${ }^{2}$ Coexistence of antibodies to factor VIII and peripheral nerve myelin has been demonstrated in a patient with chronic inflammatory polyradiculoneuropathy complicated by a coagulation disorder. ${ }^{4}$ The present association of GBS with a bleeding disorder due to ITP, as described in our case, has only been reported once previously. ${ }^{5}$

O COMBARROS J CALLEJA

L HERNÁNDEZ JM POLO Services of Neurology and Clinical Neurophysiology,

University Hospital "Marqués de Valdecilla",
Santander, Spain

Correspondence to: Dr Berciano.
1 Karpatkin S. Autoimmune thrombocytopenic purpura. Semin Hematol 1985;22:260-88.

2 Hughes RAC, Winer JB. Guillain-Barré syndrome. In: Matthews WB, Glaser G, eds. Recent advances in clinical neurology, Vol 4. Oxford: Blackwell, 1984:19-49.

3 Koski CL, Chou DKH, Jungalwala FB. Antiperipheral nerve myelin antibodies in Guillain-Barre syndrome bind a neutra glycolipid of peripheral myelin and cross1989;84:280-7.

4 Berger JR, Rosenfeld WE, Sheremata WA, et al. Chronic inflammatory polyradiculoneuropathy complicated by factor VIII antibody. Neurology 1983;33:1224-6.

5 Gross PT. Acute idiopathic polyneuritis and idiopathic thrombocytopenic purpura. JAMA 1980;243:256-7.

Isolated cerebellar syndrome: atypical form of cerebral malaria

The neurological manifestations of malaria are usually associated with the febrile attack, their outstanding features being the seizures and an impaired state of consciousness. We report a case of isolated cerebellar syndrome, a more benign complication of malaria, not related to the febrile attack.

A 31 year old French man, with no past medical history was admitted on 21 March 1989 at another hospital for a rapidly progressive ataxia. Four months previously, the patient worked as a member of the French Cooperation Group in Burkina Faso, and did not take regular prophylactic anti-malaria drugs. In January 1989, the patient had an attack of fever $\left(40^{\circ} \mathrm{C}\right)$, headaches, vomiting and diarrhoea, which was diagnosed as malaria and treated successfully with chloroquine. On 15 March 1989, he experienced rapidly progressive dysarthria and an unsteadiness on walking. A week later, the patient was referred to our hospital.

On admission, he was afebrile, but appeared chronically ill and complained of severe fatigue. There was a recent history of weight loss. Cardio-pulmonary and abdominal examinations were normal. There was no rash or lymphadenopathy. Neurological examination revealed a cerebellar syndrome interfering with a normal gait, and a less severe bilateral cerebellar ataxia. There was no abnormality of the cranial nerves, neither was there any nystagmus, or motor or sensory deficit. Tendon reflexes were present bilaterally, but were more prominent on the right side.

The following laboratory studies were normal: complete blood count, erythrocyte sedimentation rate, electrolytes, glucose, blood urea nitrogen, liver function tests, electrophoresis of plasma proteins and amylase. Serological tests for HIV1, HIV2, syphilis, Epstein Barr virus, cytomegalovirus, hepatitis B surface antigen, herpes simplex virus 1 were negative. A radiograph of the thorax, and the cerebral CT scan and MRI were normal. Blood and urine cultures were negative. The CSF was clear, under normal pressure, with six lymphocytes, and a normal glucose concentration. Protein was $0.7 \mathrm{~g} / \mathrm{L}$ and the gamma globulin count $18 \%$ with polyclonal banding. Bacteriological studies of the CSF were negative. The EEG showed diffuse slow waves, suggesting an encephalopathy.

During the following days, while there was no fever, the patient became icteric, and developed a hepatosplenomegaly confirmed by echography. There was also a pan- cytopenia. Peripheral blood films were positive for plasmodium parasites and an indirect immunofluorescent test was positive at $1 / 2560$, suggesting falciparum species. Treatment by mefloquine produced a rapid and complete recovery of the hepatosplenomegaly, pancytopenia and icterus. The cerebellar ataxia improved at the same time. The gait returned to normal two months later. Subsequent blood films for plasmodium were negative.

Even though the so called "cerebellar syndrome" is a well known but uncommon clinical presentation of malaria, ' there are few reported cases in the neurological literature. Lemercier $e t a l^{2}$ found a transient cerebellar syndrome in two of three patients presenting with severe febrile attacks, but they were usually less prominent than the other general and neurological signs of the attack. Nevertheless, the authors insisted on the frequency of the lesions involving the cerebellum, or its connections on neuropathological examination. Our patient had some common features with the 12 cases from Sri Lanka reported by Senanayake: ${ }^{3}$ cerebellar ataxia, sometimes associated with nystagmus, occurring in a febrile patient during the 15 days following a febrile attack of falciparum malaria. Gametocytes were present in the blood of these patients and the cerebellar signs subsided one to three months after the antimalarial treatment was begun. In the 12 patients the delayed onset of the neurological deficit, the absence of general signs (splenomegaly was present in one patient) and the presence of gametocytes on repeated blood smears (four out of 12 patients) suggested an immuno-allergic mechanism rather than a direct toxic event due to the plasmodium, even though there was a complete neurological recovery.

Our patient had different clinical features from those of the cases reported from Sri Lanka, but were similar to those reported by Girard et $a l^{4}$ and Garin et al. ${ }^{5}$ Clinical and laboratory findings in our patient make a viral or toxic cause unlikely.

PCHAINE F WOIMANT ORABIANU $M$ HAGUENAU Department of Neurology, Hôpital Lariboisiere, Hopital Pariboisiere,
75010 Paris, France

1 Castellani A, Chalmers AJ. Manual of tropical medicine, 2nd ed. London: Bailliere Tindall and Cox, 1913.885.

2 Lemercier G, Bert J, Nouhouayi A, et al. Coll. Le neuropaludisme: aspects électro-encépLe neuropaludisme: aspects electro-encep-
halographiques, neuro-pathologiques, halographiques, neuro-pathologiques,
problèmes physiopathologiques. Path Biol problèmes physion

3 Senanayake I. Delayed cerebellar ataxia: a new complication of falciparum malaria? $B M J$ 1987;294:1253-4

4 Girard PM, de Broucker T, Fryer D, et al. Cerebellar syndrome in mild Plasmodium falciparum malaria. Trans Roy Soc Trop Med Hygiene 1988;82:204.

5 Garin JP, Monier P, Mojon M, et al. Coll. Syndrome cérébelleux au cours d'un Girard-Lyon-S.P.C.M. Editions, 1976.

\section{A case of childhood Kufs' disease}

Kufs' disease is a form of neuronal ceroidlipofuscinosis (NCL), characterised by progressive epilepsy and dementia with motor 